\title{
Serology of Treponema pallidum infection: methodological considerations and seroprevalence in selected Italian populations
}

\author{
Claudio Galli', Pierpaolo Petasecca Donati ${ }^{2}$ per il GISSIL ${ }^{3}$ \\ 'Direzione Scientifica, Abbott Diagnostici, Roma; Laboratorio Analisi, \\ 2Istituto Dermopatico dell'Immacolata, Roma; \\ ${ }^{3} G r u p p o$ Italiano per lo Studio della Sierologia dell'Infezione Luetica (GISSIL):
}

Piero Borzini, Laura Mazzucco

Immunoematologia e Medicina Trasfusionale,

ASO "SS Antonio e Biagio"Alessandria;

Maurizio Soli, Alessandra Mengoni

Centro Immunotrasfusionale ULSS 5 Ovest Vicentino,

Ospedale di Arzignano, Arzignano (VI);

Giuseppe Fiore, Michele Ferrante

Servizio di Immunoematologia e Trasfusione,

Ospedale "Di Venere", AUSL BA4, Bari Carbonara;

Michele Scelsi, Michela Di Loreto

Servizio di Immunoematologia e Trasfusione,

Ospedale San Paolo AUSL BA4, Bari;

Roberto Cevenini, Alessandra Moroni,

Antonella Marangoni

Settore di Batteriologia Speciale e Sierologia, U.O.

Microbiologia, Policlinico S. Orsola, Bologna;

Gianluca Gessoni, Luigi Penzo

Servizio di Medicina di Laboratorio,

Ospedale Civile, Chioggia (VE);

Raffaele Borsotti, Roberta Ambrosini,

Walter Solaro

Centro Trasfusionale, Ospedale S. Biagio, Domodossola (VB);

Riccardo Serafini, Cristina D'Amico

U.O.C. Medicina Trasfusionale, ASL Frosinone,

Osp. Civile di Frosinone, Frosinone;

Adolfo Moretti, Raffaella Vannucchi

Servizio di Immunoematologia e Medicina Trasfusionale,

A.O. della Versilia, Lido di Camaiore (LU);

Giorgio Marchiori

Servizio di Immunoematologia e Trasfusione,

Ospedale Civile, ULSS I2, Mestre (VE);

Elda Suigo', Bianca Osnaghi', Helios Recalde',

Laura Galli', Elena Riccardi'

'U.O. Laboratorio Analisi e Microbiologia,

${ }^{2}$ U.O. Centro Trasfusionale e Immunoematologia, A.O.
Ospedale Civile di Legnano, Presidio Magenta-Abbiategrasso, Magenta (MI);

Giovanni Gesu, Luisa Grassi

S.C. Microbiologia e Virologia, A.O.

Ospedale Niguarda Ca' Granda, Milano;

Fernanda Dorigatti, Laura Soldini, Marcello

Marinelli

Laboraf - Immunologia e Sierologia. DIBIT 2, Milano;

Fernando Ravagnani, Arabella Mazzocchi

Servizio di Immunoematologia e Medicina Trasfusionale,

Istituto Nazionale dei Tumori, Milano;

Marisa De Palma, Chiara Vecchi, Monica De Maria

Servizio di Immunoematologia e Medicina Trasfusionale,

A.O. Policlinico, Modena;

Luigi Vigorè, Roberto Vezzo

Microbiologia, A.O. S. Gerardo, P.O. S. Gerardo dei Tintori,

Monza (MI);

Salvatore Formisano, Enzo Perna

Immunoematologia e Medicina Trasfusionale, A.O.U.

Federico II, Napoli;

Giovanni Pellegrini, Giovanna Moscato

Lab. Analisi Chimico-Cliniche e Microbiologiche, A.O.

Universitaria Pisana, Ospedale di Cisanello, Pisa;

Pierpaolo Petasecca Donati,Angela Dore

Lab. An., Ist. Dermopatico dell'Immacolata (IDI) IRCCS,

Servizio di Immmunometria, Roma;

Giuseppe Pistolese, Emilia Vaccaro

Servizio Immunotrasfusionale,

A.O. OO.RR. S. Giovanni di Dio e Ruggi d'Aragona, Salerno;

Claudio Velati, Laura Fomiatti, Lorella Baruffi

Servizio di Immunoematologia e Medicina Trasfusionale,

A.O. della Valtellina e Valchiavenna, Sondrio;

Claudio Galli

Abbott Diagnostici, Roma.

Key words: Syphilis, serology, epidemiology, enzyme immunoassays, Architect

Sierologia dell'infezione da Treponema pallidum: considerazioni metodologiche e sieroprevalenza in popolazioni italiane selezionate

\section{SUMMARY}

An increase of syphilis incidence in recent years has been reported throughout Europe and also in Italy. The advent of automated serological assays for the detection of anti-Treopnema pallidum antibodies increases the sensitivity and the reproducibility of antibody detection: one of such methods (the chemiluminescent Architect 
Syphilis TP assay) has been evaluated in a multisite study, that involved 22 Italian laboratories, on 62,036 serum specimens obtained from blood donors $(n=44,884)$, from hospital in- or outpatients at low $(n=16,482)$ or high $(n=284)$ risk for treponemal infection and from 386 patients already diagnosed with syphilis at different stages. The Architect Syphilis assay showed a good reproducibility both on controls (7I8 replicates, CV of $14.4 \%$ on negative and of $12.7 \%$ on positive control) and on clinical specimens ( 93 samples, CV of $3.61 \%$ ). The sensitivity on syphilis cases was $99.73 \%$ compared to $99.43 \%$ by other EIAs, and the specificity on 44,898 blood donors was 99.67\%. The positive predictive value was related to the signal of the Architect assay (4.3\% for sample to cutoff $(\mathrm{S} / \mathrm{CO})$ values $<3$ versus $71.8 \%$ for $\mathrm{S} / \mathrm{CO} \geq 3$ ). The positivity rates for anti-Treponema pallidum antibodies were $0.15 \%$ in blood donors, $3.46 \%$ among low risk subjects and $16.90 \%$ among patients at high risk for syphilis infection. The high prevalence in low risk groups enhances the need for a surveillance of syphilis, that appears to increase in the general population, with sensitive first-level assays. An appropriate algorithm for confirmation of low positive results by Western blot testing should be envisioned.

Received January 16, 2008

Accepted July I, 2008

\section{INTRODUZIONE}

L'incremento dei casi di sifilide segnalato sin dalla metà degli anni ' 90 nell'Europa orientale (17) si è successivamente verificato anche nei paesi industralizzati dell'Ovest europeo. Le segnalazioni provenienti dai sistemi di sorveglianza hanno infatti mostrato delle curve epidemiche molto simili in tutti gli stati: al decremento considerevole registrato dalla metà degli anni ' 80 ("effetto AIDS") è seguito, dalla fine degli anni '90, un incremento di 2-6 volte dei nuovi casi di lue, soprattutto a carico della popolazione maschile, sia omo- che eterosessuale $(2,10-12)$. In particolare, un recente "report" tedesco (2) ha evidenziato un raddoppio nella segnalazione dei nuovi casi dal 2001 al 2003, con aumento sensibile soprattutto dei casi di sifilide secondaria e latente, oltre ad un maggiore aumento delle segnalazioni, per certi versi sorprendente, nelle aree rurali rispetto alle aree urbane. Anche in Italia il sistema di sorveglianza delle malattie sessualmente trasmissibili (MST), attivo ormai da oltre quindici anni, ha segnalato un analogo incremento; più in particolare uno studio effettuato presso il Centro MST dell'Università di Milano ha mostrato dal 2000 al 2002 un aumento di quasi 5 volte dei nuovi casi (sifilide primaria, secondaria e latente precoce, cioè $<12$ mesi), in grande maggioranza nella popolazione omosessuale (4).

La diagnosi di sifilde primaria o secondaria si basa classicamente su criteri clinici e anamnestici, supportati dalla ricerca diretta del treponema nelle lesioni per i casi primari. Nelle altre forme di infezione la possibilità di identificare le lesioni viene meno, e quindi la progressiva distanza temporale tra il momento dell'infezione ed il momento diagnostico fa sì che si ricorra sempre più frequentemente all'impiego dei test sierologici, sia nelle forme secondarie tardive che, ancor più nelle forme latenti. La possibilità di impiegare sia dei test treponemici, cioè basati sull'impiego di antigeni di Treponema pallidum e sul loro riconoscimento da parte degli anticorpi del paziente, che $\mathrm{i}$ test reaginici, legati a reattività non parassita-specifiche presenti prevalentemente nelle fasi attive dell'infezione, aumenta il ventaglio di possibilità diagnostiche. Una recentissima segnalazione inglese (13) ha permesso di appurare come nei casi di infezione primaria e secondaria recente $i$ test di più frequente impiego siano soprattutto gli immunoenzimatici (EIA), nelle varie formulazioni ormai disponibili, che risultavano impiegati nel $90 \%$ dei casi.

L'ulteriore progresso nella sierologia dell'infezione luetica è rappresentato dall'impiego di test immunoenzimatici con rilevazione differente dai classici sistemi colorimetrici e disponibili su sistemi automatizzati: in questo studio abbiamo inteso valutare le caratteristiche di prestazione di un nuovo test automatizzato in chemiluminescenza nella "routine" diagnostica di Laboratori italiani e verificare la prevalenza di anticorpi antiTreponema pallidum in popolazioni a diverso rischio di infezione.

\section{MATERIALI E METODI}

Presso 22 laboratori in 8 regioni italiane (14 Centri trasfusionali, 3 Microbiologie e 5 Laboratori analisi) sono stati analizzati campioni di routine ottenuti da:

1) donatori di sangue;

2) soggetti a basso rischio per infezione luetica, intendendo come tali i pazienti interni ed esterni sottoposti ad analisi sierologiche di routine in assenza di fattori di rischio noti e/o di chiare indicazioni cliniche;

3) soggetti ad alto rischio per infezione luetica, vale a dire pazienti afferenti a centri specializzati per malattie sessualmente trasmissibili (MST) o con altre patologie infettive a trasmissione sessuale, come ad esempio l'HIV; 4) casi clinici di sifilide.

Tutti i campioni di siero ottenuti da questi soggetti sono stati analizzati con il test Architect Syphilis TP, un metodo automatizzato in chemiluminescenza che utilizza 3 antigeni ricombinanti di 
T. pallidum (TpN15, TpN17, TpN47) sulla fase solida e un coniugato anti-IgG e IgM umane. Il segnale chemiluminescente viene confrontato con un valore soglia; i campioni con un rapporto di intensità del segnale rispetto al cutoff $(\mathrm{S} / \mathrm{CO})$ uguale o superiore a 1 sono considerati reattivi per anticorpi anti-Treponema pallidum. Su diverse migliaia di campioni sono stati utilizzati in parallelo con il test Architect anche altri test treponemici (TP), sia immunoenzimatici (Murex ICE, Bouty EIA, Dsx BEIA, Diesse EIA, Diasorin Liaison) che di agglutinazione (TPHA/TPPA) o non treponemici (NT: VDRL, RPR). Su buona parte dei campioni reattivi con Architect e/o con altri test di primo livello sono stati impiegati dei dosaggi di approfondimento: FTA-Abs e Western blot. Per quest'ultimo dosaggio sono stati impiegati tre diversi metodi, tutti basati sull'impiego di antigeni ricombinanti:

a) un test "home made" già validato in precedenza (16), che utilizza sulle striscie il lisato totale proteico di T. pallidum;

b) il test Inno-LIA, che utilizza quattro antigeni ricombinanti (TpN47, TpN17, TpN15 e TmpA) e tre bande di controllo (5);

c) il test Arnika, che impiega sia gli antigeni treponemici p47, p44,5, p17 e p15 che antigeni lipoidei (VDRL) e consente di caratterizzare la risposta IgG e IgM, con l'impiego di due differenti coniugati anti-immunoglobuline umane.

Per questi tre test sono stati utilizzati i rispettivi criteri di positività indicati dal produttore, che richiedono sostanzialmente la presenza di bande di reattività contro due o tre diversi antigeni treponemici. In accordo con le linee guida esistenti (8), i campioni reattivi con almeno due test treponemici sono stati considerati positivi per anticorpi, mentre i campioni che sono risultati reattivi con il solo test Architect Syphilis sono stati considerati "falsi positivi", tranne quelli prelevati da pazienti con diagnosi clinica di sifilide e quelli ottenuti da pazienti ad alto rischio sui quali si è ottenuta una reattività ripetuta con segnale elevato.

Oltre alla sieroprevalenza nelle varie categorie ed alla concordanza tra metodi è stata anche valutata la ripetibilità del test Architect, sia sui controlli accessori del test che su campioni clinici.

\section{RISULTATI}

Popolazione. Sono stati raccolti complessivamente 62036 campioni di routine, di cui 44884 da donatori di sangue periodici o occasionali, 16482 da soggetti a basso rischio (nella maggior parte pazienti ospedalizzati o ambulatoriali), $284 \mathrm{da}$ pazienti ad alto rischio e 386 da pazienti luetici. Per questi ultimi, la caratterizzazione dello stadio di infezione era nota in 283 casi $(73.3 \%)$ : di que- sti, $7(2.47 \%)$ avevano un'infezione primaria, 97 (34.28\%) un'infezione secondaria, 161 (59.01\%) un'infezione latente, $3(1.06 \%)$ una sifilide terziaria, $6(2.12 \%)$ una neurosifilide e $3(1.06 \%)$ una infezione luetica congenita.

Caratteristiche analitiche del test Architect Syhphilis. La ripetibilità sui controlli è stata valutata su 718 repliche del controllo negativo e del controllo positivo del test, eseguite presso 11 Centri con lotti diversi di reagente. Il coefficiente di variazione totale è risultato del $14.4 \%$ per il controllo negativo (media del segnale: $0.08 \pm 0.01$ $\mathrm{S} / \mathrm{CO}$ ) e del $12.7 \%$ sul controllo positivo (media del segnale: $2.63 \pm 0.33 \mathrm{~S} / \mathrm{CO}$ ). Sui campioni inizialmente reattivi al test Architect la ripetizione con lo stesso metodo ha confermato la positività: l'analisi di 93 sieri ha mostrato anche in questo caso una elevata ripetibilità, con $\mathrm{CV}$ medio del $3.61 \%$ (range: $0.38 \%-22.71 \%$ ).

La sensibilità del test Architect sui 386 casi documentati di infezione luetica è risultata del $99.74 \%$ (385/386), con intervallo di confidenza al $95 \%$ tra $99.23 \%$ e $100 \%$. Il campione negativo con Architect era non reattivo anche con un altro test EIA. La specificità è stata valutata sulla popolazione a minore prevalenza di infezione e di più elevata consistenza numerica, cioè i 44884 donatori di sangue: la percentuale complessiva di reattività con il test Architect era dello 0.61\%, con differenze anche notevoli (tra $0.24 \%$ e $1.11 \%$ ) tra i 16 Centri che avevano analizzato questo tipo di soggetti, e la specificità è risultata del $99.67 \%$, con intervallo di confidenza al $95 \%$ tra $99.61 \%$ e $99.73 \%$ (figura I). Il valore predittivo positivo (VPP) del test Architect Syphilis in questa categoria era del $29.6 \%$; è stata anche analizzata la differenza nella predittività in ragione dell'intensità del segnale del test Architect (figura I): il VPP è risultato del $4.3 \%$ sui campioni con rapporto $\mathrm{S} / \mathrm{CO}$ inferiore a 3 e del $71.8 \%$ sui campioni con rapporto $\mathrm{S} / \mathrm{CO}$ uguale o superiore a 3 (chi quadro: $\mathrm{p}<0.005$ ). Un'altra caratteristica rilevante per un test di primo livello è la distribuzione dei risultati negativi rispetto alla soglia, indice della robustezza del test: l'analisi del segnale di 34678 campioni negativi ha messo in evidenza una media S/CO di $0.08 \pm 0.05$ (18 deviazioni standard dalla soglia) con 52 risultati "borderline" $(0.15 \%)$, compresi tra 0.90 e $0.99 \mathrm{~S} / \mathrm{CO}$; il $99^{\circ}$ percentile dei risultati negativi corrispondeva ad un valore $\mathrm{S} / \mathrm{CO}$ di 0.54 (figura II).

Infine, la concordanza qualitativa tra Architect Syphilis e gli altri test immunoenzimatici, valutata su 7844 campioni ottenuti da tutte le popolazioni in studio, era complessivamente del $99.50 \%$ (7805/7844), e variava tra il $99.39 \%$ ed il $99.91 \%$ senza differenze significative tra metodi. Il confronto numericamente più cospicuo era con il test 
Diasiron Liaison, un altro dosaggio in chemiluminescnza (figura III), e aveva dato luogo alla concordanza più elevata (2.107 campioni su 2.109, pari al 99.91\%). Un campione ottenuto da un paziente con diagnosi di sifilide latente è risultato negativo con ambedue $\mathrm{i}$ metodi $\mathrm{e}$ con RPR e debolmente positivo con TPHA (1/80), mentre due campioni erano risultati falsamente reattivi con entrambi i metodi e due solamente con Architect. Per quanto riguarda il confronto con gli altri test, la discrepanza di maggior rilievo si è osservata su due campioni che sono risultati negativi per IgG anti-Treponema pallidum con il test Diesse EIA, mentre erano fortemente positivi con Architect e con Western blot IgG (Inno-LIA, 4 bande), positivi con un test reaginico e positivi anche per IgM specifiche con un altro test EIA.

Prevalenza di anticorpi antiTreponema pallidum. La frequenza di positività tra $i$ donatori di sangue era dello $0.15 \%$; tra.i soggetti a basso rischio la prevalenza era invece del $3.46 \%$ e tra i casi ad alto rischio del $16.90 \%$ (figura IV).

Tutti i pazienti luetici erano positivi per anticorpi con uno o più test sierologici: in dettaglio, i test treponemici avevano sensibilità equivalente nelle forme primarie e secondarie e nelle forme latenti (Architect lue: $100 \%$ e $99.4 \%$ ), mentre i test reaginici avevano una sensibilità del $96.2 \%$ nelle forme primarie e secondarie e solo del $17.4 \%$ nelle latenti (figura V).

Tra gli altri test, i dosaggi EIA avevano una sensibilità comparabile al test Architect (97.9\% nelle forme primarie e secondarie, $99.4 \%$ nelle forme latenti), mentre i test di agglutinazione (TPHA/TPPA) mostravano una sensibilità rispettivamente del $94.7 \%$ e del $94.2 \%$ nei due gruppi. Le maggiori discrepanze tra $i$ vari test venivano evidenziate nelle forme latenti, come esemplificato nell'analisi delle reattività in donatori di sangue, valutata con 5 metodi diversi in uno dei Centri (figura VI). L'analisi mediante Western blot ha messo in evidenza

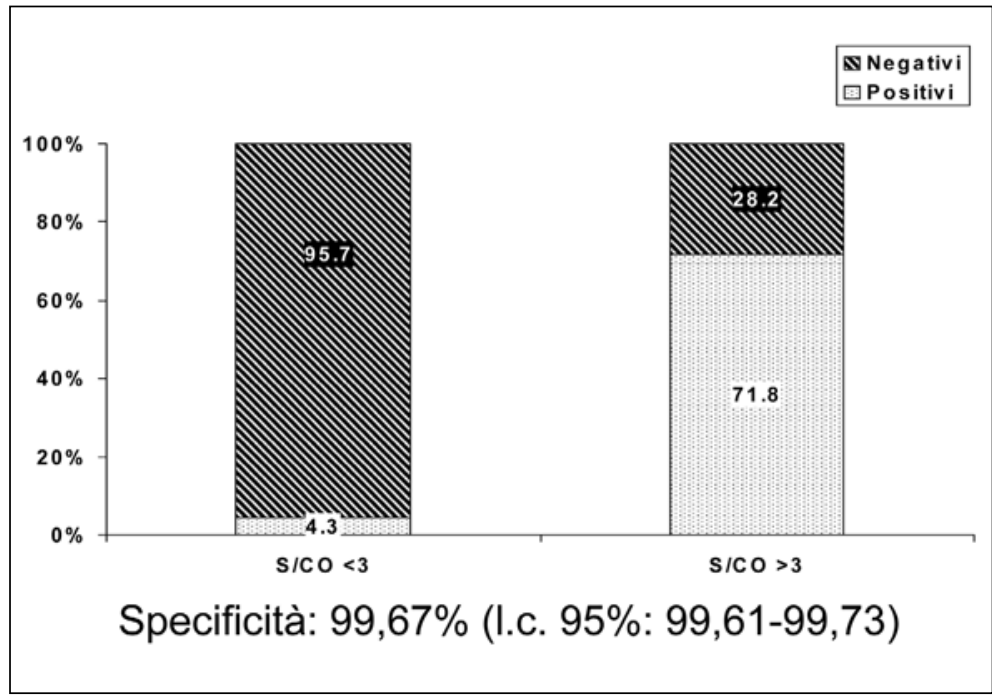

Figura I. Specificità sui donatori di sangue e valore predittivo positivo (VPP) del test Architect Syphilis a seconda dell'intensità del segnale, misurato come rapporto tra campione e valore soglia (S/CO). La differenza tra il VPP sui campioni con S/CO inferiore a 3 e uguale o superiore a 3 era statisticamente significativa (chi quadro: $p<0.005)$.

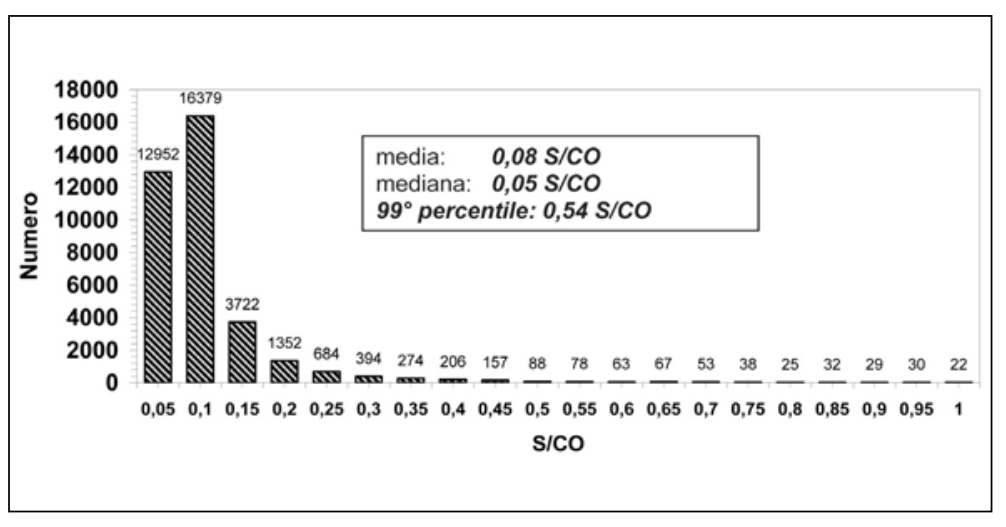

Figura II. Distribuzione dei risultati negativi (rapporto campione/cutoff, o S/CO) con il test Architect Syphilis TP su 34378 campioni di siero ottenuti da donatori di sangue. La media del segnale è situata a 18 deviazioni standard dalla soglia, i risultati "borderline" (tra 0.90 e 0.99 S/CO erano 52, pari allo $0.15 \%$ ).

Tabella I. Confronto tra i due test automatizzati in chemiluminescenza Abbott Architect e Diasorin Liaison. Sono stati analizzati in parallelo 2109 campioni di siero di routine, ottenuti da donatori di sangue, soggetti a basso o ad alto rischio di infezione e pazienti luetici. La concordanza complessiva era del 99.91\% (2 I07/2 I09) ed era anche rilevabile una discreta correlazione $(r=0.847)$ tra $i$ valori campione/cutoff (S/CO) ottenuti sui 47 campioni positivi per anticorpi antiTreponema pallidum con ambedue i metodi.

\begin{tabular}{|c|c|c|c|c|}
\hline & & & \multicolumn{2}{|l|}{ DiaSorin } \\
\hline & & Positivi & Negativi & Totale \\
\hline & Positivi & $47 \S$ & $2^{*}$ & 49 \\
\hline Architect & Negativi & 0 & $2,060 \#$ & 2,060 \\
\hline & Totale & 47 & 2,062 & 2,109 \\
\hline
\end{tabular}

$$
\begin{aligned}
& \S 2 \text { falsi positivi; * falsi positivi; \# } 1 \text { falso negativo } \\
& \text { Specificità Liaison }=99,90 \% \\
& \text { Specificità Architect }=99,81 \%
\end{aligned}
$$




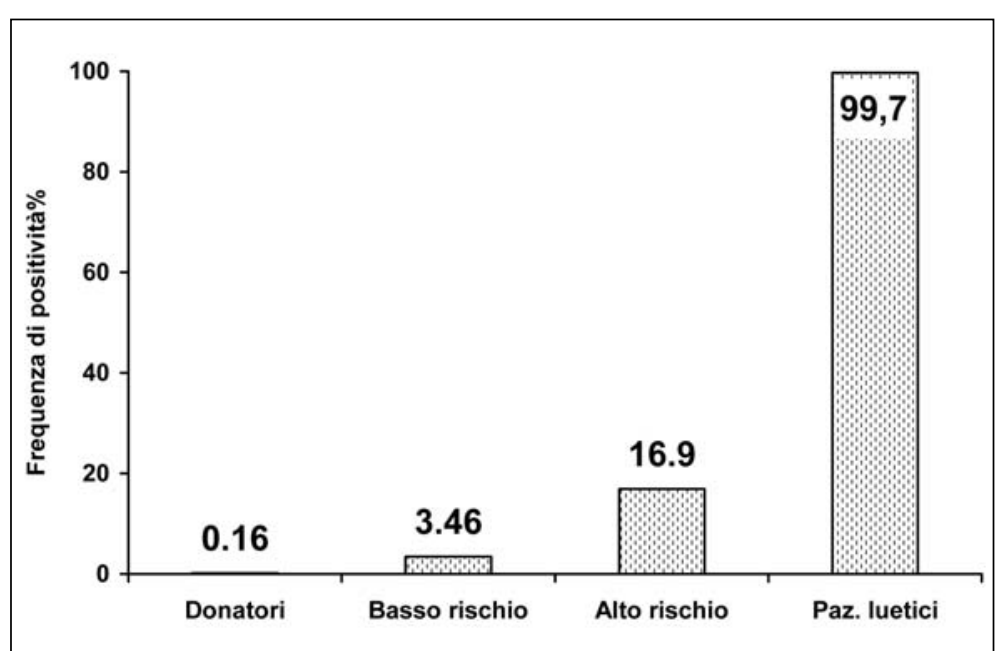

Figura III. Frequenza di positività per anticorpi anti-Treponema pallidum (test Architect Syphilis, positività verificate con altri metodi) in popolazioni a diverso rischio di infezione ed in pazienti con diagnosi di sifilide a vari stadi.

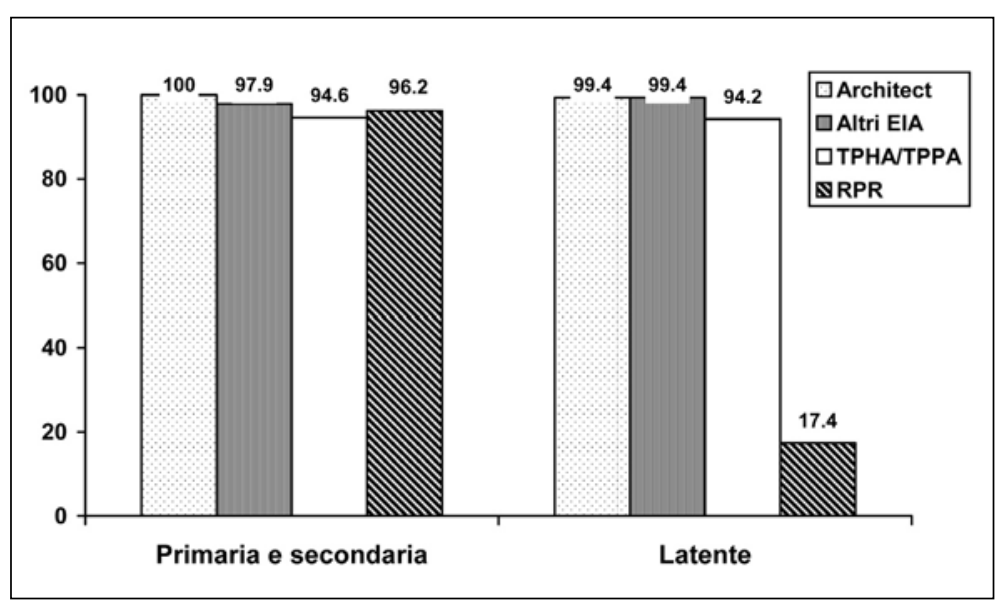

Figura IV. Sensibilità di differenti test treponemici (Architect Syphilis, altri EIA, TPHA/TPPA) e dei test reaginici su pazienti luetici a differenti stadi della malattia.

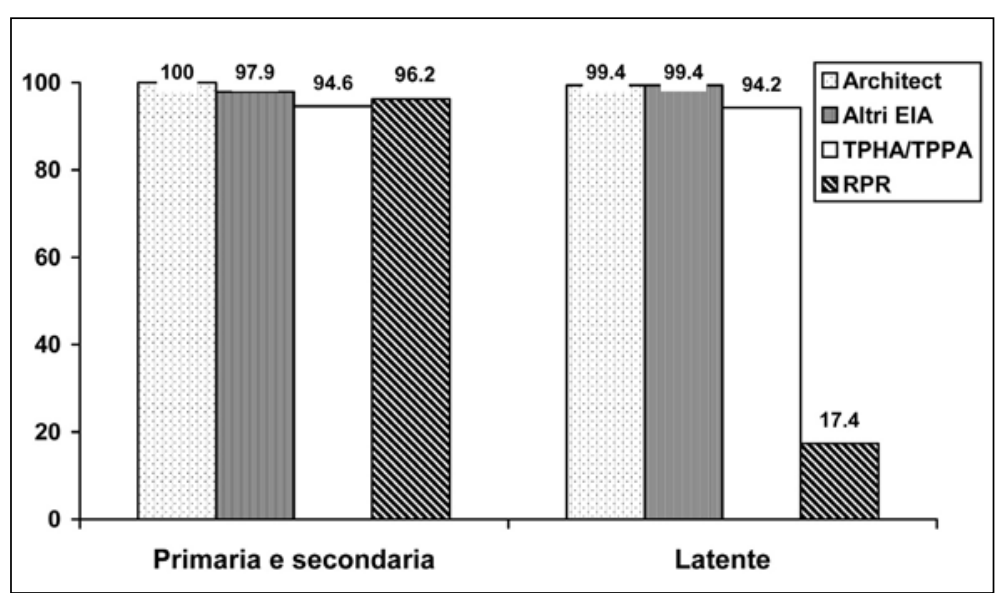

Figura V. Sensibilità di differenti test treponemici (Architect Syphilis, altri EIA, TPHA/TPPA) e dei test reaginici su pazienti luetici a differenti stadi della malattia.

prevalentemente delle reattività $\mathrm{IgG}$, rivolte con maggiore frequenza nei confronti degli antigeni TpN47 e TpN17, mentre una reattività IgM era di riscontro assolutamente occasionale.

\section{DISCUSSIONE}

I dati scaturiti da questo studio confermano l'elevata sensibilità dei test treponeimici immunoenzimatici, già indicata nello studio di McClean, et al (13). Infatti, solamente uno dei casi di sifilide è risultato non reattivo con Architect , mentre due altri pazienti erano negativi per IgG specifiche con un diverso metodo EIA commerciale e presentavano una debole reattività con un test reaginico.

Gli altri test treponemici "classici" hanno anch'essi mostrato una buona sensibilità; differente è la situazione per quanto riguarda i test reaginici, il cui impiego è risultato frequentissimo nel già citato "report" inglese (13) nella diagnosi di sifilide primaria o recente (oltre il 95\% complessivamente), con una diversa distribuzione per VDRL (68\%) e RPR (38\%). Nella nostra esperienza la sensibilità relativa di questi test nelle forme primarie è risultata adeguata, come segnalato da altri Autori $(9,13)$, ma nelle forme latenti non raggiungeva il $20 \%$, come del resto atteso dal momento che queste reattività non sono caratteristiche delle forme inattive di infezione.

La sempre maggiore diffusione dei test EIA, di semplice esecuzione e in gran parte automatizzabili, è stata accompagnata da circa un decennio da un uso sempre più frequente di antigeni ricombinanti, che rappresentano le regioni maggiormente immunogene del "mosaico" antigenico di T. pallidum, in luogo di antigeni parassitari purificati. La caratterizzazione degli antigeni maggiori (6) sia proteici (TpN17, TpN37, TpN47) che lipoproteici (TpN15, TpN44,5, quest'ultimo comune peraltro anche ad altre specie di Treponema) ha portato alla loro progressiva adozione sia per i test di primo livello (18) che, più recentemente, per gli immunoblot da utilizzare in seconda battuta per una conferma della reattività e per un a migliore caratterizzazione della risposta immune nelle varie fasi dell'infezione $(9,16)$. Le differenti opzioni per la diagnosi sierologica di lue sono state tra l'altro analizzate nelle recenti linee guida 


\begin{tabular}{|c|c|c|c|c|c|}
\hline $\begin{array}{c}\text { Architect } \\
(\mathrm{S} / \mathrm{CO})\end{array}$ & RPR & TPHA & FTA - Abs & WB & Classificazione \\
\hline 1,05 & NEG & NEG & n.d. & p47 IgG neg IgM & IND \\
\hline 1,07 & NEG & NEG & n.d. & Neg IgG e IgM & Falso positivo \\
\hline 1,14 & NEG & NEG & n.d. & p47 IgG neg IgM & IND \\
\hline 1,19 & NEG & NEG & n.d. & Neg IgG e IgM & Falso positivo \\
\hline 1,33 & n.d. & n.d. & NEG & p47 IgG neg IgM & IND \\
\hline 1,65 & NEG & NEG & n.d. & Neg IgG e IgM & Falso positivo \\
\hline 1,66 & deb. positivo & NEG & NEG & Neg IgG e IgM & Falso positivo \\
\hline 1,84 & NEG & NEG & NEG & n.d. & Falso positivo \\
\hline 2,10 & NEG & POS 1:320 & NEG & Neg IgG e IgM & Falso positivo \\
\hline 5,75 & n.d. & POS & POS 1:10 & Pos IgG (2 Ag) neg IgM & Vero positivo \\
\hline 6,38 & deb. positivo & NEG & POS 1:10 & Pos IgG (2 Ag) neg IgM & Vero positivo \\
\hline 7,61 & deb. positivo & NEG & POS 1:10 & p17 IgG neg IgM & Vero positivo \\
\hline 10,20 & deb. positivo & NEG & POS 1:10 & Pos IgG (3Ag) p47 IgM & Vero positivo \\
\hline 12,92 & NEG & POS 1:1280 & POS 1:10 & Pos IgG (3 Ag) neg IgM & Vero positivo \\
\hline 16,13 & n.d. & NEG & POS 1:10 & Pos IgG (3 Ag) neg IgM & Vero positivo \\
\hline 21,54 & deb. positivo & POS: 1:2560 & POS 1:20 & Pos IgG (3 Ag) neg IgM & Vero positivo \\
\hline
\end{tabular}

Figura VI. Analisi della reattività anticorpale anti-Treponema pallidum con 4 metodi diversi su campioni di donatori di sangue analizzati presso il SIT dell'Azienda Sanitaria Locale di Frosinone. Da rilevare la scarsa frequenza di positività in RPR e la presenza di 3 falsi negativi TPHA su campioni positivi con tre altri test treponemici, tra cui il Western blot.

europee per le diagnosi e terapia delle malattie sessualmente trasmissibili (8), nelle quali viene raccomandato di privilegiare, a scopo diagnostico, l'impiego di test treponemici di elevata sensibilità, e di valutare la eventuale reattività iniziale con un altro test treponemico, preferibilmente di diverso formato.

Diversa è la situazione dello "screening" per sifilide, che in Italia come in molti altri paesi è obbligatorio su tutte le donazioni di sangue. In questa popolazione selezionata ben difficilmente sono presenti segni o sintomi di infezione luetica, nè d'altro canto sono diffusi i fattori di rischio classici per MST; cionondimeno, anche in questa categoria si è verificato in Italia un sensibile aumento sia della prevalenza (nuovi donatori) che soprattutto dell'incidenza (donatori periodici) negli ultimi anni. Dei recenti dati nazionali (18) indicano infatti un'incidenza di 6 casi per 100000 nel 2001 e di ben 14 casi per 100000 nel 2003, e il "trend" ha mostrato un incremento costante dal 1999, anno nel quale venivano segnalati meno di 4 nuovi casi per 100000 donazioni. Ancora, i dati raccolti nella regione Lombardia nei 10 anni tra il 1996 ed il 2005 (C. Velati, comunicazione personale) hanno messo in evidenza un incremento della prevalenza da 43.5 a 105.7 per 100000 e dell'incidenza da 1.2 a 5.8 per 100000 . Questo sensibile incremento si può in parte attribuire all'utilizzo di test di screening più sensibili (EIA), riferito da alcune strutture trasfusionali (15), anche se appare difficile credere che il solo test impiegato possa rappresentare la causa principale del fenomeno. Va infatti considerato che nello stesso periodo si è registrato nei donatori periodici anche un aumento, sia pure numericamente molto più contenuto, dei casi di infezione da HIV, ed è quin- di verosimile che una minor attenzione al rischio di acquisire infezioni sessualmente trasmissibili sia alla base di entrambi i fenomeni. A supporto di questa ipotesi il dato che a livello nazionale e in Lombardia rispettivamente per il $51.8 \%$ e il $61 \%$ dei casi di positività per lue non veniva indicato alcun fattore di rischio, mentre prevedibilmente il $37.2 \%$ ed il $31.3 \%$ dei donatori sieropositivi per $T$. pallidum indicava come fattore di rischio i rapporti sessuali, generalmente occasionali.

La prevalenza di anticorpi anti-T. pallidum nella popolazione a basso rischio analizzata in questo studio appare abbastanza elevata, suggerendo che vi sian una maggiore diffusione dell'infezione nella popolazione generale anche in Italia. Come tra i donatori di sangue, anche in questa categoria di soggetti la maggior parte dei casi di sieropositività per lue è rappresentata da casi di infezione latente e non sono quasi mai identificabili o dichiarati dei fattori di rischio, il che rende estremanente difficile sia l'identificazione della causa di infezione nei singoli soggetti che, ancor più, una ricostruzione delle potenziali epidemie "nascoste". Questa situazione ha delle implicazioni considerevoli in ambito sanitario: il significato delle recenti epidemie di sifilide infatti non risiede unicamente negli elevati livelli di morbidità causati da questa infezione, ma anche nel potenziale impatto sulla disseminazione dell'infezione da HIV. Vì è infatti una concreta evidenza che la sifilide, come pure altre MST, aumenta significativamente la trasmissione sessuale di HIV (1), e del resto nella recente osservazione sul picco epidemico di lue in soggetti omosessuali segnalato a Milano (4) oltre il 25\% dei pazienti con sifilide recente erano HIV positivi. È stato ipotizzato che la maggiore incidenza di sifilide sia il portato di 
variazioni nelle abitudini sessuali, nel senso di una maggiore promiscuità e, soprattutto, di una minore percezione del rischio di infezioni trasmissibili. Non vi sono ancora degli studi sistematici sulle variazioni nei comportamenti e nelle attitudini sessuali, ma sono identificabili diversi cambiamenti che potrebbero aver influito sull'emergere delle nuove infezioni luetiche. Tra di essi, i viaggi internazionali con maggiori opportunità di incontri sessuali in gran parte mercenari e, in alcuni paesi e regioni, la crescita della prostituzione da strada, che coinvolge anche popolazioni in età infantile ed adolescenziale. In particolare, l'aumento degli scambi commerciali tra Est ed Ovest ha causato una rapida penetrazione degli aspetti commerciali legati al sesso nei primi, con variazioni profonde nei costumi e nello stile di vita. Soprattutto tra i giovani, l'enfasi sulla scelta individuale, la celebrazione della sessualità e la diffusione di immagini a sfondo sessuale ha causato un profondo cambiamento nei costumi, senza un'adeguata preparazione e informazione sanitaria (1). Per contro, la massiccia immigrazione in occidente dai Paesi dell'Europa dell'Est ha portato ad un incremento della prostituzione da strada, che coinvolge anche gli e le adolescenti. Queste variazioni possono avere un impatto negativo sulla diffusione dell'epidemiologia di tutte le infezioni sessualmente trasmissibili, incluso l'HIV, nel continente europeo e in particolare in Italia, e induce ad una maggiore sorveglianza, che richiede da parte dei Laboratori l'adozione di strategie di screening e diagnosi in cui l'accuratezza complessiva della determinazione anticorpale deve partire dalla scelta di presidi diagnostici di adeguata sensibilità.

Da un punto di vista operativo, la sensibilità del nuovo test immunoenzimatico che abbiamo valutato appare elevata, e quindi in grado di identificare sia le infezioni luetiche recenti che le forme latenti e le "cicatrici" sierologiche indicative di una pregressa infezione, trattata e superata. Il significato clinico di una positività per anticorpi anti-Treponema pallidum in questi ultimi casi è scarso, mentre rimane rilevante l'utilità di questa informazione a livello epidemiologico $(12,15$, 17). La specificità del test Architect Syphilis, valutata nella popolazione dei donatori, è apparsa adeguata ed equivalente all'atteso (99.67\%): ovviamente, il dato di maggior rilevanza pratica per la gestione del risultato del test è il valore predittivo positivo (VPP) che, dipendendo sia dalla specificità del metodo che dalla sieroprevalenza, è basso nelle popolazioni a basso rischio: nella nostra esperienza, il VPP nei donatori di sangue è risultato del $29.6 \%$, e di circa $1^{\prime} 80 \%$ nei soggetti a basso rischio. L'evidenza, peraltro attesa, di una correlazione significativa tra intensità del segnale e positività "vera" per anticorpi anti-Treponema pallidum suggerirebbe anche in questo campo l'adozione di un algoritmo che tenga conto, più che della situazione clinica, purtroppo spesso non segnalata al momento dell'invio del campione in laboratorio, di questo parametro analitico, analogamente a quanto è stato recentemente suggerito nell'ambito dei test sierologici anti-HCV (3). Tale algoritmo potrebbe consistere nell'effettuazione di almeno un altro test treponemico nei casi che risultino positivi a basso livello con il test Architect, privilegiando a tal fine gli immunoblot, che presentano delle caratteristiche di modulazione della risposta maggiormente adatte per una refertazione accurata. Il significato di una positività isolata con un test di screening per anticorpi anti-Treponema pallidum, che in base a quanto sopra enunciato andrebbe considerata una "falsa positività", merita tuttavia un approfondimento: se da un lato è vero che per qualsiasi test di primo livello sono individuabili delle false reattività "biologiche", cioè legate a condizioni di interferenza caratterstiche di alcuni individui e non presenti nella maggioranza della popolazione, è altresì importante ricordare che anche nelle popolazioni a basso rischio o a rischio non noto queste reattività possono indicare un'infezione pregressa. Quest'ultima ipotesi trova un interessante riscontro in uno studio australiano (14), che analizzando i risultati reattivi con un test EIA ad elevata sensibilità non confermati con altri metodi aveva documentato come questi risultati fossero molto più frequenti in pazienti afferenti a centri MST. L'evidenza di una reattività più persistente con il test EIA era fornita da tre pazienti, di cui uno aveva sierorevertito con almeno altri due test, tra cui FTA-Abs e TPPA, e si presume che lo stesso fosse avvenuto anche per gli altri due. Nelle conclusioni degli Autori, un risultato positivo isolato con un test EIA particolarmente sensibile deve quindi stimolare ulteriori indagini per una infezione luetica pregressa.Un'ultima osservazione riguarda la maggiore frequenza di positività per lue nel sesso maschile, già menzionata nelle diverse osservazioni epidemiologiche $(2,4,10$, 11, 12, 17): oltre a queste, una recente analisi effettuata su donatori scozzesi (7) aveva posto l'accento sulla maggiore frequenza di positività in donatori maschi, correlata inoltre con l' età (45.2\% sopra i 44 anni contro $12.7 \%$ sotto i 44 anni). Nell'esperienza dello studio GISSIL la frequenza di reattività iniziale per sesso rifletteva la maggiore consistenza numerica della popolazione maschile, che rappresentava il $72 \%$ di tutti i donatori e il $76 \%$ dei soggetti reattivi, ma tra i positivi "confermati" i maschi rappresentavano ben l' $83 \%$ 
dei casi, a riprova dell'attenzione dovuta a questo dato di laboratorio che può indicare la presenza di comportamenti a rischio, attuali o pregressi, anche in una popolazione selezionata.

\section{BIBLIOGRAFIA}

1. Birley H, Duerden BI, Hart CA (eds.). Sexually Transmitted Diseases: microbiology and management. $J$ Med Microbiol 2002; 51: 793-807.

2. Brown AE, Sadler KE, Tomkins SE, et al. Recent trends in HIV and other STIs in the United Kingdom: data to the end of 2002. Sex Transm Infect 2004; 80: 159-66.

3. Centers for Disease Control and Prevention. 2003. Guidelines for laboratory testing and results reporting of antibody to hepatitis C virus. MMWR 2003; 52 (RR-3): 1-15.

4. Cusini M, Ghislanzoni M, Bernardi C, Carminati G, Zerboni R, Alessi E. Syphilis outbreak in Milano, Italy. Sex Transm Infect 2004; 78: 154.

5. Ebel A, Vanneste L, Cardinaels M, et al. Validation of the INNO-LIA syphilis kit as a confirmatory assay for Treponema pallidum antibodies. J Clin Microbiol 2000; 38: 215-9.

6. Fujimura K, Ise N, Ueno E, Hori T, Fujii N, Okada M. Reactivity of recombinant Treponema pallidum (r-Tp) antigens with anti-Tp antibodies in human syphilitic sera evaluated by ELISA. J Clin Lab Analysis 1997; 11: 315-322.

7. Galea G, Dow BC. Comparison of prevalence rates of microbiological markers between bone/tissue donations and new blood donors in Scotland. Vox Sang 2006; 91: 28-33.

8. Goh BT, van Voorst Vader PC. European Guideline for the management of syphilis. In: Radcliffe KW, van Voorst Vader PC, Ross JDC, et al, eds. European sexually transmitted infections management guidelines (IUSTI/WHO). Int J STD AIDS 2001; 12(Suppl 3): 14-26.

9. Goh BT. Syphilis in adults. Sex Transm Infect 2005; 81: 448-452.

10. Halslos AM, Edgardh K. An outbreak of syphilis in Oslo. Int J STD AIDS 2002; 13: 370-372.

11. Isakbaeva E, et al. Surveillance for Syphilis in Sweden. Eurosurveillance 2005; 3: 11-13.

12. Markus U, Bremer W, Hamouda O. Surveillance for syphilis in Germany. Eurosurveillance 2004; 10: 10-14.

13. McClean H, Daniels D, Carne C, Bunting P, on behalf of the National Audit Group of the British Association for Sexual Health and HIV. UK national audit of early syphilis management. Case notes audit: diagnosis and treatment. Int J STD AIDS 2006; 17: 334-339.

14. Ooi C, Robertson P, Donovan B. Investigation of isolated positive syphilis enzyme immunoassay (ICE Murex) results. Int J STD AIDS 2002; 13: 761-764.

15. Regine V, Piccinini V, Gonzalez M, Catalano L, Hassan HJ. Sorveglianza delle malattie infettive trasmissibili con la trasfusione (SMITT) nell'anno 2003. Notiziario ISS 2006; 19 (2): 3-8.

16. Sambri V, Marangoni A, Eyer C, et al. Western Immunoblotting with five Treponema pallidum recombinant antigens for serologic diagnosis of syphilis. Clin Diagn Lab Immunol 2001; 8 (3): 534-539.

17. Tichonova L, Borisenko K, Ward H, Meheus A, Gromyko A, Renton AM. Epidemics of syphilis in the Russian Federation: trends, origins, and priorities for control. Lancet 1997; 350: 210-213.

18. Young H, Moyes A, Seagar L, McMillan A. Novel recombinant-antigen enzyme immunoassay for serological diagnosis of syphilis. J Clin Microbiol 1998; 36 (4): 916-917. 